\title{
New MAC Protocol for Fast Data Collection in Wireless Sensor Networks
}

\author{
Shabana V V, Shiju Kumar P S \\ ${ }^{*}$ Pursuing MTech, Department of Computer Science and Engineering, University of Calicut \\ MES College of Engineering, Kuttipuram, India \\ "Assistant Professor, Department of CSE MES College of Engineering, Kuttipuram, India
}

\begin{abstract}
Wireless sensor network (WSN) is a powerful combination of distributed sensing, computing and communication. These are deployed in number of scenarios, and in most cases, the data collection rate plays the crucial role. The key application scenario of wireless sensor networks is data gathering: sensor nodes transmit data, possibly in a multi-hop fashion, to an information sink. The performance of sensor networks is thus characterized by the rate at which information can be aggregated to the sink. In this paper, after studying the existing approaches, we are looking for an effective protocol, to enhance the rate of data collection in wireless sensor networks. We are introducing a new MAC protocol using asynchronous TDMA. In conventional TDMA protocol, if a device has no data to send during a particular time slot, that slot is wasted. Also, if data is of larger size then it cannot be transmitted in the particular slot. Here, these two problems are addressed and appropriate solutions are encountered by making use of this new protocol.
\end{abstract}

Keywords: - Convergecast, TDMA scheduling, multiple channels, power control, asynchronous TDMA protocol.

\section{INTRODUCTION}

Wireless Sensor Networks (WSNs) have become an established technology for a large number of applications, ranging from monitoring (e.g., pollution prevention, precision agriculture, structures and buildings health) to event detection (e.g., intrusions) and target tracking (e.g., surveillance).WSNs usually consist of a large number of sensor nodes, which are battery-powered tiny devices.

A sensor is a device that can be controlled and queried by an external device to detect, record, and transmit information regarding a physiological change or the presence of various chemical or biological materials in the environment. A collection of such sensors form a network that can be used for efficient monitoring of health, environment, military etc. A wireless sensor consists of a small processor, memory, power, sensing and transceiver units. Additionally, a sensor can have location finding system, mobilize and a power generator which are application dependent sub-units. The size and weight of a sensor limits the processing capability, amount of memory and the amount of power that it can store. A major part of power consumed by a sensor is used to run the transceiver circuitry. As, the transmission range of a sensor increases the power consumed by the transceiver also increases. Since they have limited transmission range sensors together form a multi-hop radio network to accomplish communication amongst themselves

\section{RELATED WORKS}

A number of techniques have been applied so far for enhancing the data collection rate. Minimizing the schedule length by using orthogonal codes or hopping sequences to get rid of interference is studied by Annamalai [1], where they consider assigning different time slots and code pair to interfering links. They proposed an algorithm CCTCAA which constructs a tree with schedules assigned to nodes for collision free convergecasting. The algorithm is capable of code allocation in case multiple codes are available, to minimize the total duration required for convergecasting.

A closely related study by Gandham [2] focuses on finding a TDMA schedule that minimizes the total time required to complete a convergecast in WSN. Their aim was to guarantee a bound on convergecast latency. They propose a scheduling algorithm that requires at most $3 \mathrm{~N}$ time slots where $\mathrm{N}$ is the number of nodes in the network. They were interested in showing the bounds on the schedule length with different network organizations. In this work non-aggregated version of the convergecast problem has been considered by Gandham in the presence of a single channel and TDMA protocols, where the goal is to minimize the schedule length.

Another study by Moscibroda [3] shows that in sensor networks even if node placement is worst case a high rate can be maintained. The theoretically achievable worst-case rate in sensor networks remains high even as network size grows. They advocate studying scaling laws that capture the achievable rate in worst-case, i.e., 
arbitrarily deployed sensor networks. This novel notion of worst-case capacity concerns the question of how much information can each node transmit to the source, regardless of the network's topology.

A later work on enhancing data collection rate by O.D. Incel and B. Krishnamachari [4] focused on methods that can reduce the schedule length by eliminating the limitations due to interference and connectivity. They described a realistic simulation-based study on tree-based data collection. They took advantage of utilizing transmission power control, multiple frequencies, and in addition proposed efficient routing topologies. It is shown that once all the interfering links are removed by use of multiple frequencies, the data collection rate becomes limited by the maximum degree of the tree. They also showed that this rate can further be increased on degree-constrained trees.

A closely related work by A. Ghosh, O.D. Incel, V.S.A. Kumar, and B. Krishnamachari [5] focus on the link scheduling problem of maximizing the aggregated data collection rate at the sink node under the setting of TDMA protocols and multiple frequency channels. Their present work is different from the rest in that they proposed algorithms and proved several important theoretical results on the aggregated convergecast problem under multiple frequencies and also gave an upper bound on the maximum number of such frequencies required and propose a polynomial time algorithm that minimizes the schedule length under this scenario.

A more effective approach identified is the one that take advantage of all these techniques and applied hierarchically to achieve a higher data collection rate in [6] by O. Durmaz Incel, A. Ghosh, B. Krishnamachari, and $\mathrm{K}$. Chintalapudi. They elaborate on the performance of the previous work, a receiver-based channel assignment (RBCA) method, and compared its efficiency with other channel assignment methods and introduced heuristics for constructing optimal routing trees to further enhance data collection rate. Using extensive simulations, they showed that scheduling transmissions on different frequency channels is more effective in mitigating interference as compared to transmission power control and also evaluated the performance of three different channel assignment methods: 1) Joint Frequency Time Slot Scheduling (JFTSS), 2) Receiver-Based Channel Assignment (RBCA), and 3) Tree-Based Multichannel Protocol (TMCP).

The steps in its design can be summarized as to start with tree construction and then continue with interference-aware scheduling. If the nodes can control their transmission power, scheduling phase is coupled with a transmission power control algorithm. If the nodes can change their operating frequency, channel scheduling can be coupled with time slot scheduling as it is the case with the JFTSS algorithm or first channels are assigned and then time slot scheduling continues as in the case of RBCA.

However, the TMCP algorithm considers tree construction and channel assignment jointly and then does the scheduling of time slots.

\section{PROPOSED SYSTEM}

Existing work had the objective of minimizing the completion time of converge casts and had experimentally investigated the impact of transmission power control and multiple frequency channels on the schedule length. But these techniques were applied based on contention-free TDMA protocol that faces some limitations. If a device has no data to send during a particular time slot, that slot is wasted. Also, if data is of larger size then it cannot be transmitted in the particular slot. The proposed system addresses these two problems and appropriate solutions are encountered by making use of a new protocol.

The new approach that is focused in this paper is to use asynchronous TDMA based MAC protocol. This takes into account the role of power control and use of scheduling on multiple frequency channels for enhancing the data collection rate which is already used in the previous work. However these are performed based on a new MAC protocol to address the above mentioned limitations. This is achieved $\backslash$ by making use of Asynchronous TDMA where a device can start transmission in any of the slots if it is free. Also, the second problem is overcome by dividing data in to smaller packets, so that even larger packets can be passed.

Following assumptions are made regarding the functioning of the protocol.

- A tree based network in which is each node is identified by using a unique Id is considered.

- There is one sink node which is at the top of the tree as root node.

- Other nodes are leaf nodes which act as source nodes.

- Sink node supports multiple frequency reception.

This protocol has the following modules.

- Scheduling - Each source node is allotted with a time slot. Based on the topology information, two or more devices can transmit in the same slot, provided their signals will not interfere with each other.

- Receiver based multiple frequency selection: The receiver nodes in the path can decide which frequency the source node has to use for transmission. So simultaneously if two devices are transmitting, the receiver is able to receive them.

- Power control mechanism based on the following equation (1) is used for controlling the transmission power of the sender.

Pnew $=(\beta /$ SINR $) *$ Pcurrent 
Pnew : the new transmission power level in the next iteration

Pcurrent : the current transmission power level

\section{IMPLEMENTATION DETAILS}

\section{A. Initialization Steps}

- Nodes are allotted with time slots.

- Receivers in the path decide what frequencies can be used by the sources for transmission and particular frequencies are assigned for all source nodes. ( No two neighboring nodes will not have same frequency)

\section{B. Steps at Source Node}

- If any node has some data to transmit to the Sink node, it checks the slot number. If the time slot corresponds to its own, then it starts transmission of packet in that particular slot.

- If the slot does not correspond to its own time slot, then it senses the channel for a time period ' $\mathrm{t}<\mathrm{Tt}$ '. Where $\mathrm{Tt}$ is the duration of the time slot assigned for each node

- If the channel is idle for duration of ' $t$ ', then the source node starts transmission.

- If the channel is not idle, then the source node waits for its own slot.

\section{Steps at Destination Node}

- When a node receives some data, it checks the frequency of the signal. Based on this it determines the address of the sender. (note that in normal TDMA destination identifies the source based on the time slot number. And in Normal ATDMA it is identified by adding the id of the sender node in each packet that is transmitted. But here we are using a different method where, the source is identified by the frequency of the signal and so the extra overhead of adding source id in each packet is eliminated.

\section{Steps at the Sink Node}

- The sink node collects every packet from each of its leaf node and aggregates it to form the final data.

The project is simulated using NS-2 simulator. Nodes are static and are deployed in a tree structure. Transmissions occur towards the sink node. At the beginning of the simulation channel scanning is performed. IEEE 802.15.4 standard using the 2.4GHz ISM band that supports 16 Channels is used here.

\section{EXPERIMENTAL RESULTS}

The proposed system (ATDMA based MAC protocol) is compared with the existing system. Throughput, average delay and energy are used as the measurement parameters. Throughput is measured as the ratio of number of packets received at the destination per unit time. Figure 1 shows the time delay graph for both existing and proposed system. Here the average delay for collecting the data from node 9 to the sink node is considered. The graph shows that use of ATDMA based protocol reduces the delay for data collection further as compared to the TDMA based system.

Figure 2 shows the plot of remaining enegy at node 9 as the simulation time goes on. The graph shows that the energy consumption is less in the proposed wok as compared to the existing system.

Figure 3 shows the comparison of proposed wok against the existing system in terms of throughput. It shows that even though the ATDMA based system has poor throughput initially, this achieves a better throughput as the simulation time goes on. 


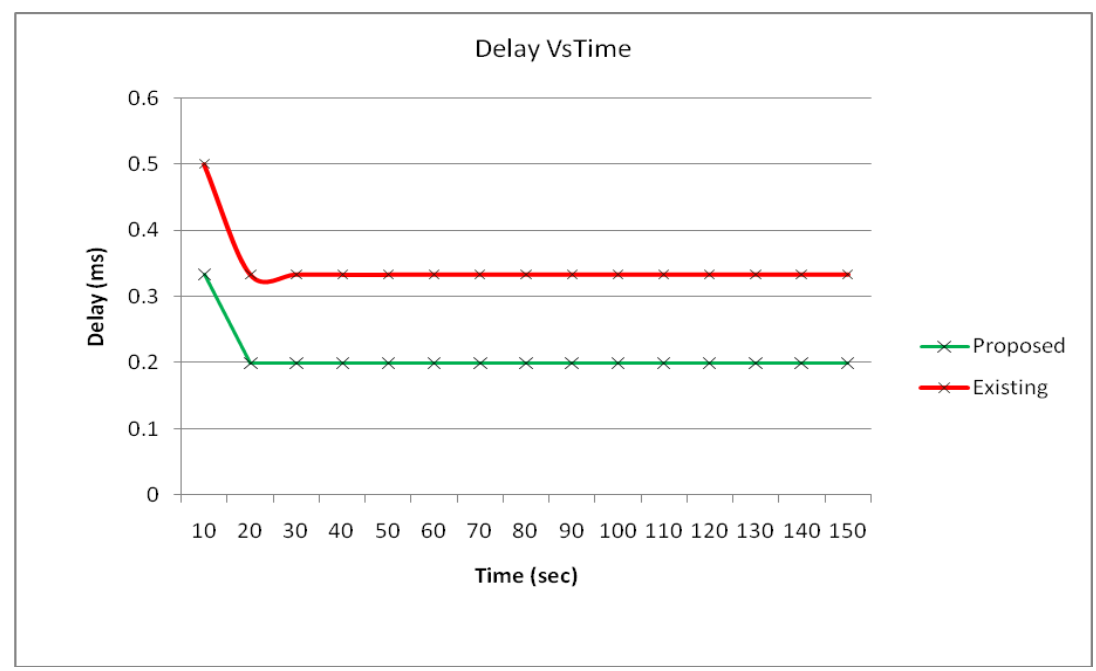

Fig 1.Comparison of existing sytem with proposed in terms of Average delay

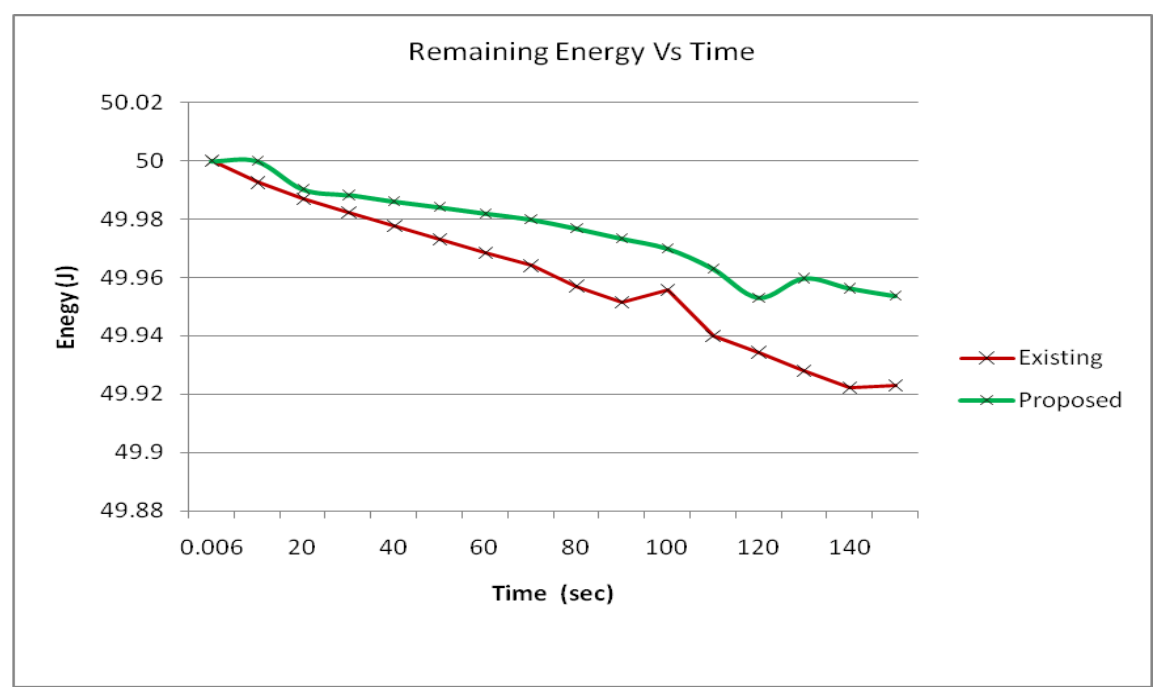

Fig 2.Comparison of existing sytem with proposed in terms of energy remaining at node 9

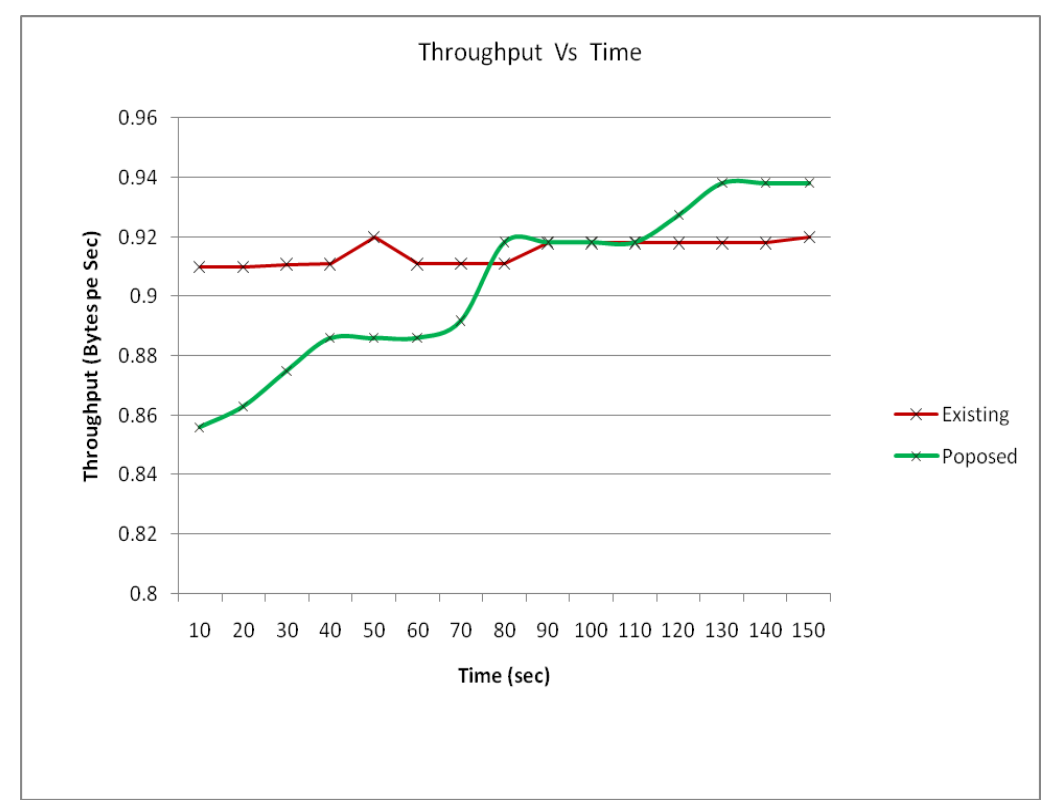

Fig 3.Comparison of existing sytem with proposed in terms of throughput 


\section{CONCLUSION}

A number of techniques are concerned so far using realistic simulation models to address the speed of data collection. These techniques possess their own advantages and disadvantages. A more effective approach identified is the one that take advantage of all these techniques and applied hierarchically to achieve a higher data collection rate. This approach was studied in detail and finally identified a problem that if a device has no data to send during a particular time slot, that slot is wasted. Also, if data is of larger size then it cannot be transmitted in the particular slot. In the proposed system these are addressed by making use of a new MAC protocol. This results in reducing the time delay for data collection further

\section{REFERENCES}

[1] I. Akyildiz, W. Su, Y. Sankarasubramaniam, and E. Cayirci, "A survey on sensor networks," Communications Magazine, IEEE, vol. 40, no. 8, pp. 102 - 114, aug. 2002.

[2] V. Annamalai, S.K.S. Gupta, and L. Schwiebert. On tree-based convergecasting in wireless sensor networks. In Wireless Communications and Networking, 2003. WCNC 2003. 2003 IEEE, volume 3, pages 1942 - 1947 vol.3, march 2003.

[3] S. Gandham, Ying Zhang, and Qingfeng Huang. Distributed minimal time convergecast scheduling in wireless sensor networks. In Distributed Computing Systems, 2006. ICDCS 2006. $26^{\text {th }}$ IEEE International Conference on, page 50, 2006.

[4] T. Moscibroda. The worst-case capacity of wireless sensor networks. In Information Processing in Sensor Networks, 2007. IPSN 2007. 6th International Symposium on, pages 1 \{10, april 2007.

[5] O.D. Incel and B. Krishnamachari. Enhancing the data collection rate of tree-based aggregation in wireless sensor networks. In Sensor, Mesh and Ad Hoc Communications and Networks, 2008. SECON '08. 5th Annual IEEE Communications Society Conference on, pages 569 -577, june 2008.

[6] A.Ghosh, O.D. Incel, V.S.A. Kumar, and B. Krishnamachari. Multi-channel scheduling algorithms for fast aggregated convergecast in sensor networks. In Mobile Adhoc and Sensor Systems, 2009. MASS '09. IEEE 6th International Conference on, pages 363 -372, oct. 2009.

[7] O. Durmaz Incel, A. Ghosh, B. Krishnamachari, and K. Chintalapudi. Fast data collection in tree-based wireless sensor networks. Mobile Computing, IEEE Transactions on, 11(1):86 -99, jan.2012.

[8] Ying Chen and Bhaskar Krishnamachari. MCC:A high-throughput multi-channel data collection protocol for wireless sensor networks. jun. 2011.

[9] Pei Huang; Li Xiao; Soltani, S.; Mutka, M.W.; Ning Xi, "The Evolution of MAC Protocols in Wireless Sensor Networks: A Survey," Communications Surveys \& Tutorials, IEEE, vol.15, no.1, pp.101,120, First Quarter 2013

[10] Arifuzzaman, M.; Matsumoto, M.; Sato, T., "An Intelligent Hybrid MAC With Traffic-DifferentiationBased QoS for Wireless Sensor Networks," Sensors Journal, IEEE, vol.13, no.6, pp.2391,2399, June 2013

[11] Prakash Rao Vaddina, Dimitri Marandin," Simulation of IEEE 802.15.4/ZigBee with Network Simulator-2 (ns-2)"available online from www.ifn.et.tu.dresden.de/ marandin/ZigBee/ZigBeeSimulation.html

[12] De Pasquale, A.; Magnani, N.P.; Zanini, P., "Interference evaluation in asynchronous TDMA cellular systems," Universal Personal Communications Record, 1997. Conference Record., 1997 IEEE 6th International Conference on, vol., no., pp.58,63 vol.1, 12-16 Oct 1997

[13] T. Salonidis and L. Tassiulas, Asynchronous TDMA: Scheduling and Performance In Technical Report TR 2002, , Institute of Systems Research (ISR), 2004, Univesity of Maryland, College Park 